\title{
Regimen-Specific Effects of RNA-Modified Chimeric Antigen Receptor T Cells in Mice with Advanced Leukemia
}

\author{
David M. Barrett, Xiaojun Liu, ${ }^{2}$ Shuguang Jiang, ${ }^{2}$ Carl H. June, ${ }^{2}$ \\ Stephan A. Grupp, ${ }^{1,3, *}$ and Yangbing Zhao ${ }^{2, *}$
}

\begin{abstract}
Cytotoxic T lymphocytes modified with chimeric antigen receptors (CARs) for adoptive immunotherapy of hematologic malignancies have demonstrated activity in early phase clinical trials. While T cells bearing stably expressed CARs are efficacious and have potential long-term persistence, temporary expression of a CAR via RNA electroporation is also potentially efficacious in preclinical models. Temporary CAR expression using RNA presents a method of testing CARs clinically with additional safety where there may be concerns about possible chronic "on-target, off-tumor" toxic effects, as the degradation of RNA ensures complete removal of the CAR over time without relying on suicide induction systems. CD19-directed RNA CAR T cells were tested in vivo for efficacy and comparison to lentiviral vector (LV)-generated stable CAR T cells. We tested the hypothesis that multiple infusions of RNA CAR T cells preceded by lymphodepleting chemotherapy could mediate improved survival and sustained antitumor responses in a robust leukemia xenograft model. The saturation strategy using rationally designed multiple infusions of RNA CARs based on multiple model iterations approached the efficacy of a stable LV expression method. Two-color imaging revealed that relapse was a locoregional phenomenon in both the temporary and the stable expression models. In marked contrast to stably expressed CARs with retroviral or LV technology, the efficacy of RNA CARs appears independent of the costimulatory signaling endodomains likely because they more influence proliferation and persistence rather than short-term efficacy. The efficacy of the RNA CAR infusions may approach that of stably expressed CARs, offer theoretically safer initial clinical testing in addition to suicide systems, and allow for rapid and effective iterative preclinical modeling for the testing of new targets.
\end{abstract}

\section{Introduction}

$\mathbf{N}$ EW METHODS ARE NEEDED to overcome the poor immunogenicity of acute lymphoblastic leukemia (ALL), and immune therapies have the potential to be efficacious with less toxicity than standard cytotoxicity approaches currently used in high-risk and relapsed disease (Horowitz et al., 1990). Chimeric antigen receptors (CARs) combine antibody-based specificity for tumor-associated surface antigens with T-cell receptor-activating intracellular domains providing specific antitumor cellular immune activity (Eshhar et al., 1993; Brocker and Karjalainen, 1998). CARs allow a T cell to achieve major histocompatibility complex-independent primary activation through single-chain $\mathrm{Fv}(\mathrm{scFv})$ antigen-specific extracellular regions fused to intracellular domains that provide T-cell activation and costimulatory signals. Cytotoxic T cells (CTLs) endowed with CARs can augment cytokine secretion and antitumor activity in a variety of solid tumor and leuke- mia models (Pinthus et al., 2004; Milone et al., 2009; Kochenderfer et al., 2010a).

Most investigators have achieved efficient CAR gene transfer into human $\mathrm{T}$ cells via retroviral or lentiviral vectors (LVs) for human tumor antigens, with some of these cell therapy products are advancing to phase I/II trials in B cell malignancies (Kershaw et al., 2006; Pule et al., 2008; Till et al., 2008, 2012; Kochenderfer et al., 2010b; Brentjens et al., 2011; Savoldo et al., 2011). Recently, our group reported the use of CD19-targeted CAR + T cells in three patients with CLL, resulting in two complete responses and one partial response followed by the report of two complete responses in pediatric ALL (Kalos et al., 2011; Porter et al., 2011a; Grupp et al., 2013). These responses have been sustained, and the CAR+ $\mathrm{T}$ cells persist for up to 24 months, suggesting the longterm efficacy of this approach. Approaches producing stable long-term expression of the CAR on infused cells across multiple cell divisions have clear advantages for long-term

\footnotetext{
${ }^{1}$ Division of Oncology and ${ }^{3}$ Department of Pathology, Children's Hospital of Philadelphia, Philadelphia, Pennsylvania 19104.

${ }^{2}$ Abramson Family Cancer Research Institute and Department of Pathology \& Laboratory Medicine, Perelman School of Medicine, University of Pennsylvania, Philadelphia, Pennsylvania 19104.

*These two authors contributed equally to this work.
} 
immunosurveillance; however, transiently expressed CARs may also have a role in clinical development.

Electroporation-mediated mRNA transfection holds promise as a complementary approach to gene expression that does not result in permanent genetic modification of cells. The use of mRNA for gene therapy applications was first described by Malone et al. (1989) in the context of liposome-mediated transfection. Transfer of TCR genes via electroporation of mRNA into primary $\mathrm{T}$ lymphocytes has been described previously (Zhao et al., 2005, 2006). Building on data in preclinical models using RNA CARs in solid tumors after intratumoral injection or in locoregional injection intraperitoneal models, we reported success using anti-CD19 RNA CARs against disseminated leukemia in a xenograft model (Rabinovich et al., 2009; Barrett et al., 2011b). This was recently repeated using mRNA electroporation of the same 19-41BB- $\zeta$ CAR into NK cells in a xenograft preclinical model of a single injection of CAR-modified NK cells (Shimasaki et al., 2012). In comparison to long-term (integrating) expression systems, mRNA transfection allows more rapid iterative changes in CAR design to move into a good medical practice (GMP)-compliant system with potentially lower cost and less complex release testing compared with lentiviral or retroviral vectors. In settings where long-term expression is required for clinical efficacy and/or repeated cell infusion not possible, the mRNA approach would not be suitable. However, for initial testing of antigens where "on-target, offtissue" toxicity (Morgan et al., 2010) might be a risk, transient expression could potentially allow for greater safety in initial clinical CAR testing while still providing efficacy, especially as CARs targeting solid tumor antigens are being tested.

CD19 is a surface antigen restricted to B cells and is expressed on early pre- or pro-B cells and on a majority of B cell leukemias and lymphomas (Nadler et al., 1983). This makes CD19 an ideal antigen for targeted therapy, as it is expressed on the malignant cell lineage and a specific subset of early and mature B lymphocytes but not hematopoietic stem cells. Although previous reports have used multiple injections, these have been largely in intraperitoneal/intratumoral models or use a single regimen without investigation of dose or schedule modification (Zhao et al., 2010). We sought to investigate specific factors (dose, timing, and lymphodepletion) that will determine the most successful strategy for the application of temporarily modified CAR T cells in a disseminated model of leukemia. We hypothesized that a multiinfusion protocol of transient anti-CD19 CAR expression via mRNA transfection optimized for these variables would provide effective adoptive T-cell therapy for leukemia comparable with a stably expressed CAR via LV transfection. We refined and optimized this approach using a robust xenograft model of CD19+ leukemia, identical to the model used for preclinical validation of the stable lenitiviral CAR used in the phase I trials recently reported (Milone et al., 2009; Kalos et al., 2011; Porter et al., 2011a; Grupp et al., 2013).

\section{Materials and Methods}

\section{Construction of in vitro transcription vectors and RNA electroporation}

CD19 and mesothelin (meso)-targeted CARs with 4-1BB and $\mathrm{CD} 3 \zeta$ signaling domains (19-BBz and ss1-BBz, respectively) have been described previously (Carpenito et al., 2009;
Milone et al., 2009). The PCR products were subcloned into a pGEM.64A-based vector by replacing GFP from pGEMGFP.64A (Zhao et al., 2006) with restriction-enzyme-digested PCR products with HindIII and NotI to produce pGEMss1.bbz.64A and pGEM-CD19bbz.64A. Similarly, thirdgeneration versions of the CARs were constructed utilizing the CD28 signaling domain. The replaced CAR cDNAs were confirmed by direct sequencing and linearized by SpeI digestion before RNA in vitro transcription. mScript RNA System (Epicentre, Madison, WI) was utilized to generate capped IVT RNA. The IVT RNA was purified using an RNeasy Mini Kit (Qiagen, Valencia, CA) and purified RNA was eluted in RNase-free water at $1-2 \mathrm{mg} / \mathrm{ml}$. Human T cells were stimulated by CD3/CD28 beads as described (Carpenito et al., 2009). The stimulated T cells were washed three times with OPTI-MEM and resuspended in OPTI-MEM at the final concentration of $1-3 \times 10^{8} / \mathrm{ml}$ before electroporation. Subsequently, the stimulated T cells were mixed with $10 \mu \mathrm{g} / 0.1 \mathrm{ml} \mathrm{T}$ cells of IVT RNA (as indicated) and electroporated in a $2 \mathrm{~mm}$ cuvette (Harvard Apparatus BTX, Holliston, MA) using an ECM830 Electro Square Wave Porator (Harvard Apparatus BTX). Viability post-transfection ranged from $50 \%$ to $80 \%$, and in all cases viable $\mathrm{T}$ cells for injection had $>99 \%$ CAR expression at the time of use. For the trafficking experiments, $\mathrm{T}$ cells were stably transduced with a click beetle red luciferase lentiviral construct before mRNA transfection.

\section{Construction of LVs}

LVs that encode the various CARs under the transcriptional control of the EF- $1 \alpha$ promoter were generated as previously described (Imai et al., 2004; Milone et al., 2009). Sequences encoding the click beetle luciferase (Pyrophorus plagiophthalamus) were inserted into the self-inactivating LV and codon optimized to emit in the green $\left(\lambda_{\text {peak }} 550 \mathrm{~nm}\right)$ or red $\left(\lambda_{\text {peak }} 630 \mathrm{~nm}\right)$ spectrum by modifying the active pocket as described (Tafreshi et al., 2008; Mao, 2011). All constructs were verified by sequencing.

\section{CAR detection on electroporated T cells}

Cells were washed and suspended in FACS buffer (phosphate buffered saline with $0.1 \%$ sodium azide and $0.4 \%$ bovine serum albumin). Cells were incubated at $4^{\circ} \mathrm{C}$ for $25 \mathrm{~min}$ with biotin-labeled polyclonal goat antimouse $\mathrm{F}(\mathrm{ab}) 2$ antibodies (anti-Fab; Jackson Immunoresearch, West Grove, PA) and then washed twice with FACS buffer. Cells were then stained with phycoerythrin-labeled streptavidin (BD Pharmingen, San Diego, CA). Flow cytometry acquisition was performed with a BD FacsCalibur (BD Biosciences), and analysis was performed with FlowJo (Treestar, Ashland, OR).

\section{Mouse xenograft studies}

Studies were performed as previously described with certain modifications (Teachey et al., 2006). Briefly, 6-10week-old NOD-SCID- $\gamma \mathrm{c}^{-/-}$(NSG) mice were obtained from the Jackson Laboratory (Bar Harbor, ME) or bred in house under an approved institutional animal care and use committee protocol and maintained under pathogen-free conditions. Animals were injected via tail vein with $10^{6}$ viable Nalm-6 or primary leukemia cells engineered to express click 
beetle green (CBG) luciferase. $\mathrm{T}$ cells were injected via tail vein at the indicated dose 5-7 days after injection of leukemia. All cells were of human origin. Animals were closely monitored for signs of xenogeneic graft-versus-host disease (GVHD; typical onset $>60$ days) and other toxicity as evidenced by $>10 \%$ weight loss, loss of fur, diarrhea, or conjunctivitis as well as for leukemia-related hind limb paralysis. Expression of the CD19 or SS1 scFv CARs was detected using the biotinylated $\mathrm{F}\left(\mathrm{ab}^{\prime}\right) 2$ fragment from goat antimouse $\operatorname{IgG}$ sera (specific for scFvs of murine origin; Jackson ImmunoResearch) followed by staining with streptavidin-PE (BD Biosciences/Pharmingen).

\section{Bioluminescent imaging}

Anesthetized mice were imaged using a Xenogen Spectrum system and Living Image v4.2 software. Mice were given an intraperitoneal injection of $150 \mathrm{mg} / \mathrm{kg}$ D-luciferin (Caliper Life Sciences, Hopkinton, MA). Previous titration of Nalm-6 and human $\mathrm{T}$ cells or primary leukemia cells transduced with the click beetle luciferase vectors indicated time to peak of photon emission to be $5 \mathrm{~min}$, with peak emission lasting for 6-10 $\mathrm{min}$, similar to our experience with firefly luciferases. Each animal was imaged alone (for photon quantitation) or in groups of up to five mice (for display purposes) in the anterior-posterior prone position at the same relative time point after luciferin injection $(6 \mathrm{~min})$. Data were collected until the midrange of the linear scale was reached (600-60,000 counts) or maximal exposure settings reached (f stop 1, large binning, and $120 \mathrm{sec}$ ), and then converted to photons $/ \mathrm{sec} / \mathrm{cm}^{2} /$ steradian to normalize each image for exposure time, f stop, binning, and animal size. For anatomic localization, a pseudocolor map representing light intensity was superimposed over the grayscale body-surface reference image. For data display purposes, mice without luciferase containing cells were imaged at maximal settings and a mean total flux value of $3.6 \times 10^{5} \mathrm{p} / \mathrm{sec}$ was obtained. Mice with luciferase-containing leukemia typically became moribund when photon flux approached $2 \times 10^{11} \mathrm{p} / \mathrm{sec}$, giving a detection range of 6 orders of magnitude. Similarly, luciferase-expressing versions of the various CARs were used to detect trafficking of the $T$ cells to tumor sites and to assess expansion of the transferred $\mathrm{T}$ cells in the host mouse. For simultaneous two-color imaging, luciferin was again administered in saturating amounts and data were collected via Living Image v4.2 over the 500$800 \mathrm{nM}$ range in $20 \mathrm{n} M$ filter increments, the spectral unmixing algorithm was applied, and then the green and red components were displayed either as individual components or as overlays (Nakajima et al., 2005; Gammon et al., 2006; Lu et al., 2009; Mezzanotte et al., 2011).

\section{Cell line identity testing}

Parent cell lines and primary leukemia samples were genotyped by short tandem repeat analysis (Masters et al., 2001). Cell lines and samples were verified every 6 months, or after any genetic modification such as CD19 or luciferase transduction to insure identity.

\section{Statistical considerations}

Analysis was performed with STATA version 10 (Statacorp, College Station, TX) or Prism 4 (Graphpad Software, La
Jolla, CA). For comparison among multiple groups, KruskalWallis analysis was performed with Dunn Multiple Comparison tests to compare individual groups. Survival curves were compared using the log-rank test with a Bonferroni correction for comparing multiple datasets.

\section{Results}

Two-color imaging reveals $T$-cell traffic to and persist at sites of disease recurrence even after losing CAR expression

As previously reported, a single infusion of $2.5 \times 10^{7}$ mRNA-engineered $\mathrm{T}$ cells results in significant disease reduction (Barrett et al., 2011b). After a period consistent with loss of the CAR because of degradation of mRNA and proliferation of $\mathrm{T}$ cells, leukemia was observed to progress. We evaluated whether the disease progression was because of leukemic harbor sites or loss of T cells by a novel two-color luciferase system to visualize the leukemia and the $\mathrm{T}$ cells simultaneously. Mice were given $10^{6} \mathrm{Nalm}-6$ expressing a variant of the CBG luciferase ( $\lambda_{\text {peak }} 550 \mathrm{nM}$ ) followed 5 days later by $10^{7}$ mRNA-transfected CAR $+\mathrm{T}$ cells, which stably expressed a red-shifted variant $\left(\mathrm{CBR} ; \lambda_{\text {peak }} 630 \mathrm{nM}\right)$ of the same click beetle luciferase. Image acquisition followed by spectral unmixing of the two luciferase signals revealed that leukemia is dramatically and quickly reduced by RNA CARs targeting CD19 but not mesothelin (Fig. 1A). The T cells were widely distributed, and there was no evidence of a resistant harbor site at this time point consistent with our previous observations in this model with single-color imaging of the leukemia (Barrett et al., 2011b). Seven days after T-cell administration, green spectrum leukemia signal was seen in the jaw and skull base, despite continued widespread persistence of $\mathrm{T}$ cells. Thirteen days after T-cell infusion, the leukemia became disseminated and the now non- or lowCAR-expressing $\mathrm{T}$ cells remained similarly distributed (Fig. 1A). RNA CARs targeted to mesothelin were also widely distributed by this time, but did not slow leukemia progression (Fig. 1B). Proliferation of the targeted CAR T cells was quantified by bioluminescent imaging, both in the presence and in the absence of target to again confirm that proliferation is not solely caused by nonspecific xenogeneic stimulation (Supplementary Fig. S1; Supplementary Data are available online at www.liebertonline.com/hum). T cells expressing an anti-CD19 CAR in the presence of target show sustained proliferation more so than in the absence of target, or $\mathrm{T}$ cells expressing a CAR for an irrelevant antigen (mesothelin) $(p<0.01)$.

\section{Depletion of previously CAR+ T cells by cyclophosphamide}

Cyclophosphamide (CTX) is an alkylating agent used as chemotherapy for leukemia and autoimmune disorders that is also an effective agent in conditioning for allogeneic transplant (Bleyer, 1985). It has been used as a lymphodepleting agent for adoptive cell transfer in a variety of clinical trials and in preclinical models, including for the specific depletion of activated T cells and/or Tregs (Bracci et al., 2007; Lee et al., 2011). We investigated the use of CTX as an agent to reduce the potentially interfering presence of CARnegative $\mathrm{T}$ cells observed in the previous single-dose study 
A

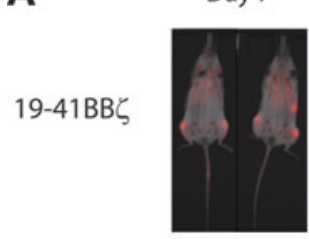

ss1-41BBC

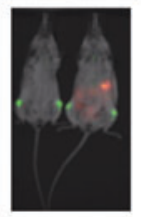

Day 13
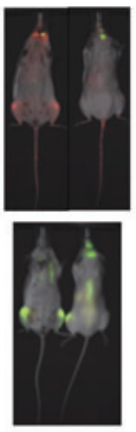

B

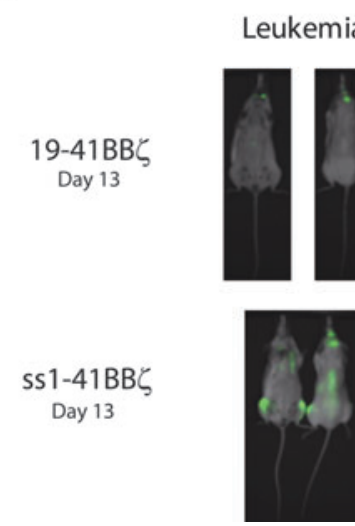

Day 18
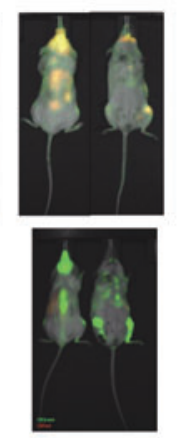

Tcells
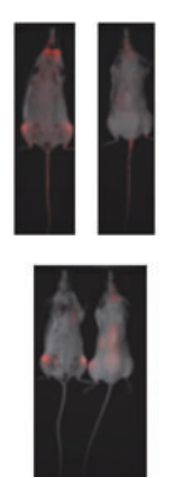

C

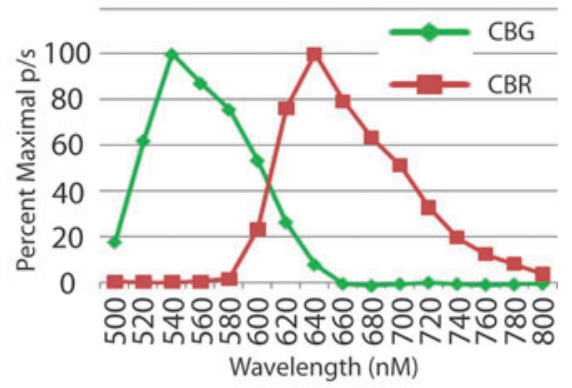

D

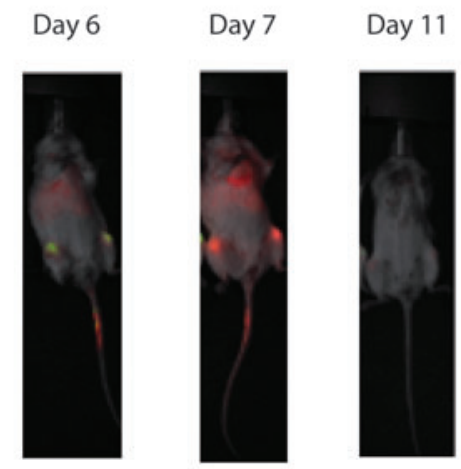

FIG. 1. Two-color imaging shows tumor response and T-cell distribution with a single injection of mRNA-engineered antiCD19 CAR T cells. (A) Time course of tumor response. NSG mice were given $1 \times 10^{6}$ CBG Nalm- 6 on day 0 , followed by $1 \times 10^{7}$ CBR CAR T cells targeting CD19 (top row) or mesothelin via the SS1 scFv (bottom row). T cells are widely distributed and the leukemia signal undetectable after 2 days (day 7). T cells with no target for the CAR are less widely distributed, and leukemia remains present. Over time, the leukemia can be seen to relapse in the skull base, jaw, and abdomen (day 13, middle panel) followed by disseminated progression. Leukemia progresses unchecked in the ss1-BBz-treated mice. (B) Single-color components of day 13 overlays from part A show that T cells are widely distributed in both CD19 and mesothelin-directed groups by this time point. Despite the co-localization in the mesothelin CAR T cells, they have no effect on leukemia progression. (C) Spectra acquired for use in unmixing from mice in the prone position. (D) CTX (60 mg/kg IP) effectively reduces transferred T cells. Mice were treated as in (A), with $1 \times 10^{6}$ CBG Nalm-6 on day 0 followed by $1 \times 10^{7}$ CBR CAR T cells targeting CD19 on day 5 . About $24 \mathrm{hr}$ after T-cell injection, leukemia is still visible in the femurs. After $48 \mathrm{hr}$, the red Tcell signal has increased and the green leukemia signal has diminished. About $24 \mathrm{hr}$ before day 11, a single dose of IP CTX is given, and imaging shows a reduction in the red signal consistent with reduction in T-cell number. There are no detectable $\mathrm{T}$ cells or leukemia cells in the peripheral blood at these time points. CAR, chimeric antigen receptor; CBG, click beetle green; CTX, cyclophosphamide; NSG, NOD-SCID $-\gamma_{\mathrm{c}}^{-/-} ; \mathrm{scFv}$, single-chain Fv.

before infusion of new RNA CAR $+\mathrm{T}$ cells. We first performed a dose titration to identify a dose that had no discernible effect on leukemia progression based on bioluminescent imaging, and determined that $60 \mathrm{mg} / \mathrm{kg}$ IP $\times 1$ (range $60-200 \mathrm{mg} / \mathrm{kg}$ ) dosing for the NSG mouse had no significant effect on survival or leukemia progression (data not shown). This is consistent with lymphodepletion dosing in other reports, and below typical effective antileukemia doses in mice (Elkin et al., 2004). Using the two-color model to simultaneously track $\mathrm{T}$ cells and leukemia, we injected mice with $10^{6}$ Nalm-6-expressing CBG luciferase (Nalm-6/CBG) on day 0 and $10^{7} \mathrm{~T}$ cells modified with CBR luciferase and electroporated with mRNA for the 19-41BB-z CAR. As observed before, the red T cells localized with the leukemia, expanding and reducing disease over the first $48 \mathrm{hr}$ from injection of T cells (Fig. 1D). A single dose of CTX administered 5 days after T-cell injection significantly reduced the signal from the transferred T cells after $24 \mathrm{hr}$ (Fig. 1D). To follow up on this observation, a larger study was undertaken with NSG mice injected intravenously with $10^{6}$ Nalm-6/CBG on day 0 followed by $10^{7}$ RNA CAR T cells on day 7. Animals were given $60 \mathrm{mg} / \mathrm{kg}$ IP of CTX on day 14 followed $24 \mathrm{hr}$ later by $10^{7}$ RNA CAR T cells. Surviving animals were given CTX again on day 27 followed by a third infusion of RNA CAR T cells on day 28. Earlier preclinical models using stably transduced CD19 CAR + T cells with retroviruses have utilized a schedule of 3 or 4 separate injections approximately weekly with $3-4 \times 10^{7}$ total T cells in similar models (Brentjens et al., 2007). There was a significant difference in both bioluminescent disease burden and survival (median OS 29 vs. 56 days, $p<0.01$ ) if CTX was given between T-cell infusions versus those that did not receive 

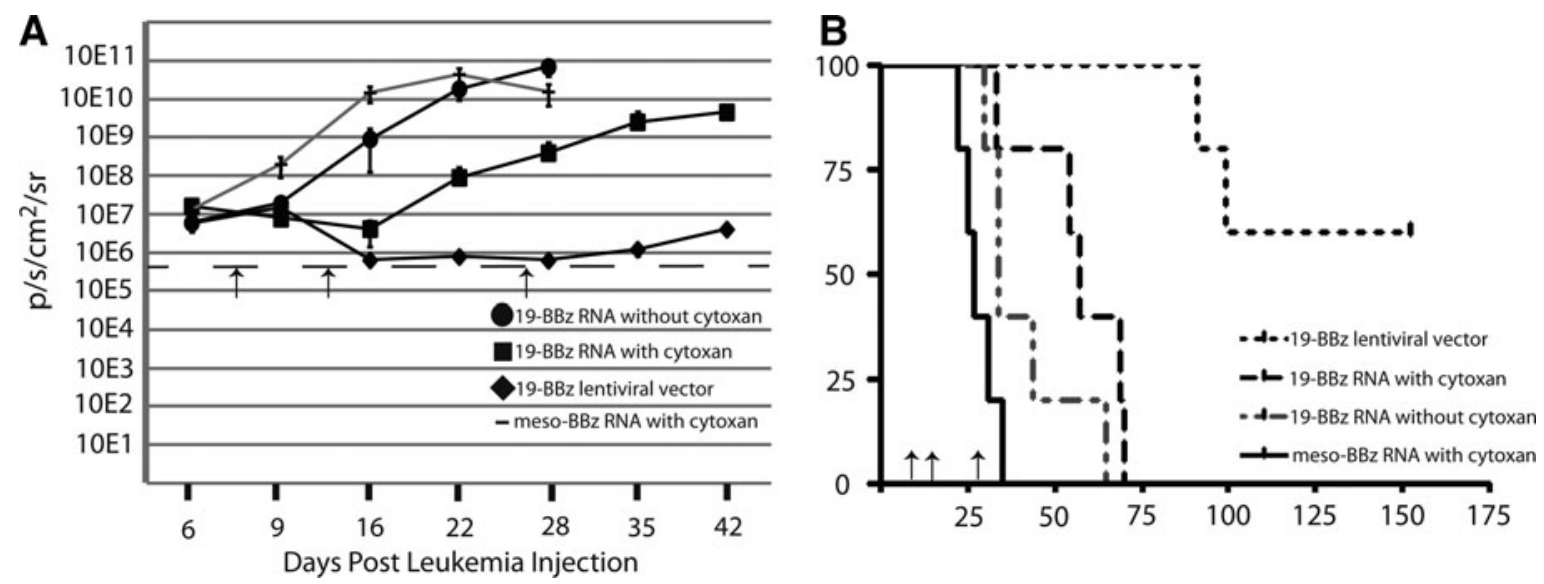

FIG. 2. Interval lymphodepletion produces a sustained antitumor effect with multiple infusions of mRNA CAR T cells. (A) CTX plus mRNA CAR T cells produce a more sustained disease control than that without CTX. NSG mice bearing Nalm-6 CBG were treated with $10^{7}$ mRNA CAR T cells on days 7, 14, and 28 with $60 \mathrm{mg} / \mathrm{kg}$ CTX IP 24 hr before injections 2 and 3 . While disease responded to the first injection of anti-CD19 CAR T cells, only with CTX did subsequent infusions have any impact on disease progression. About $10^{7}$ stably transduced CAR T cells (via lentiviral vector) produce a sustained disease response as well, and this is not lost with CTX treatment. mRNA CAR T cells directed against mesothelin with CTX do not affect disease progression. (B) The reduction in disease burden correlates with increased median survival ( $n=5$ per arm).

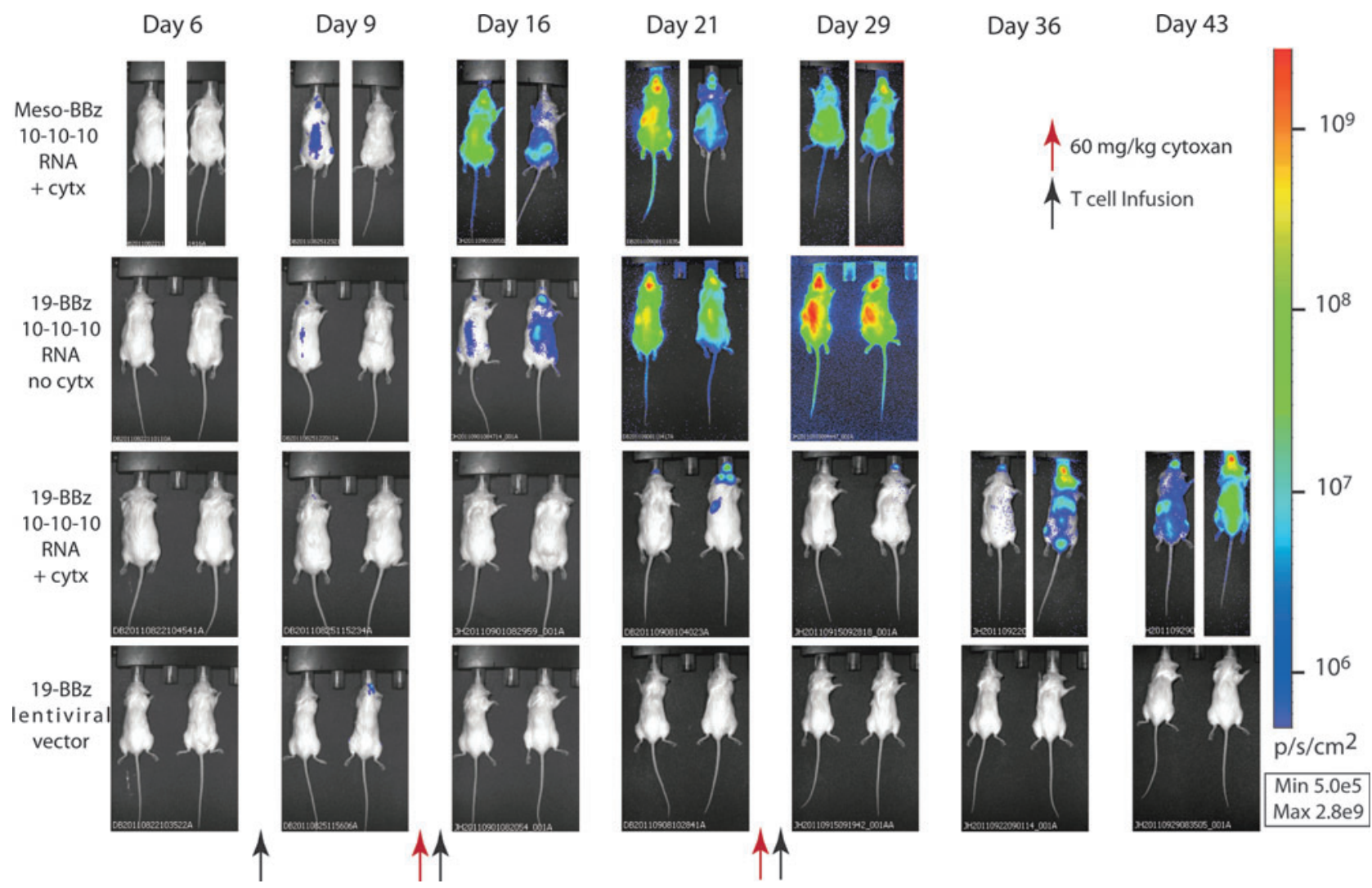

FIG. 3. Disease progression in animals treated with CTX and mRNA CAR T cells. Animals are from experiment described in Fig. 2. Mesothelin-directed T cells and cells administered without interval CTX show progression after the first injection. Repeat T-cell infusions with CTX show sustained disease response, with subsequent infusions able to impact relapsing disease (third row, change between days 21 and 29). Once infusions are stopped the disease does progress, with the exception of those mice treated with stably expressed 19-41BB-z. In the lentiviral vector 19-41BB-z animals, which received CTX on day 27, disease is eliminated quickly and no harbor sites are seen but no reduction in later xenogeneic GVHD is seen either. GVHD, graft-versus-host disease. 
A
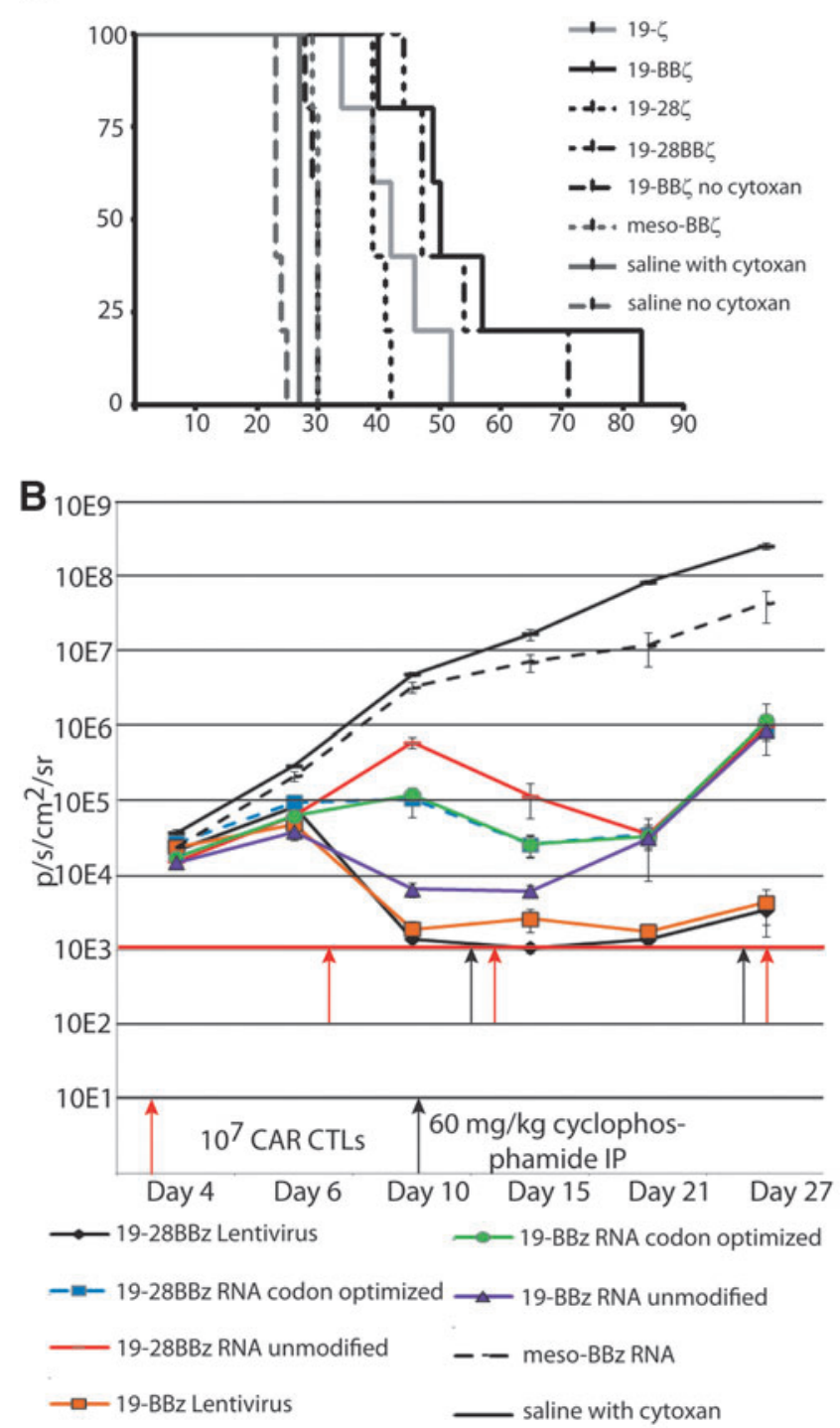

C
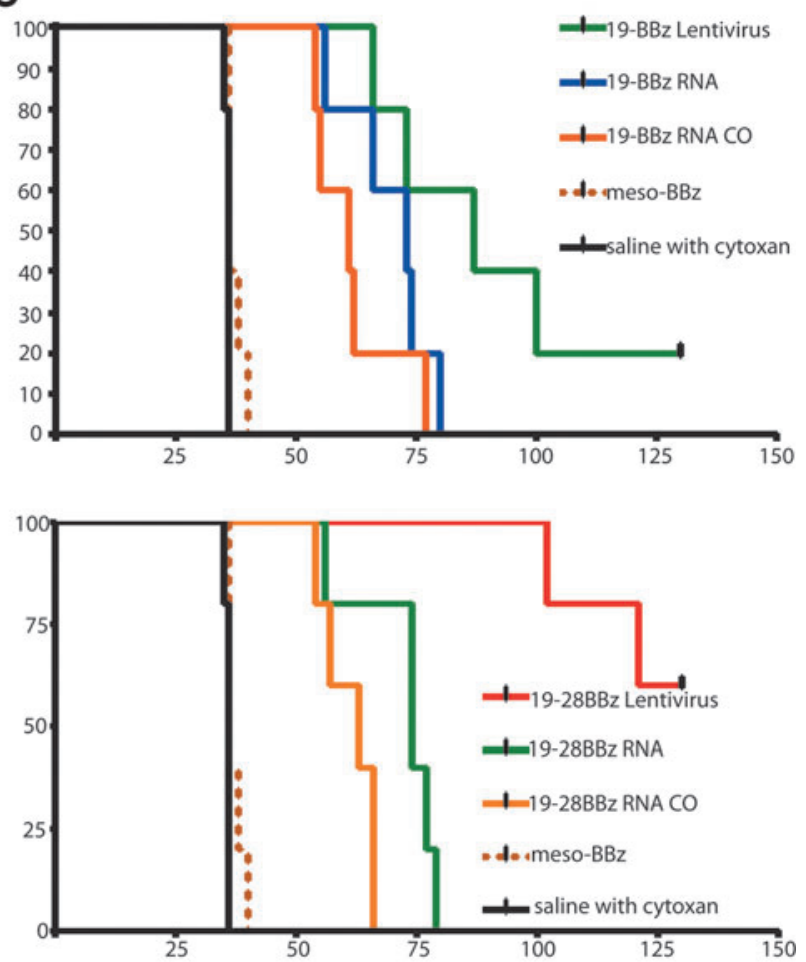

D

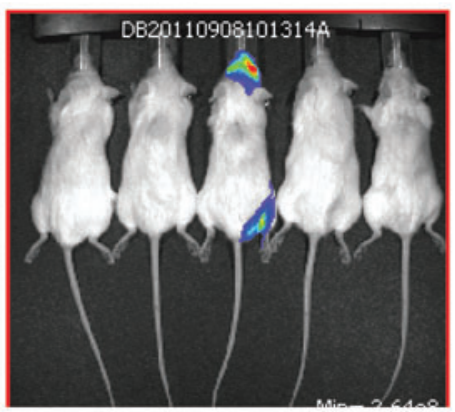

19-28BBz Lentivirus

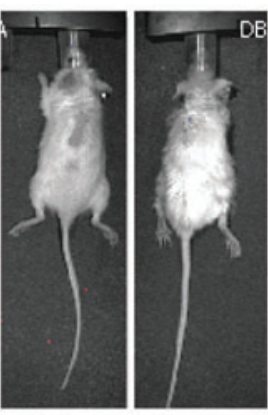

19-BBz Lentivirus

FIG. 4. (A) There is little difference among RNA CAR constructs in survival, though CTX is required for multiple infusions to have efficacy. NSG mice $\left(n=5\right.$ per experiment, repeated twice) were given $10^{6}$ Nalm- 6 CBG on day 0 followed by the 3 infusion schedule of $10^{7}$ mRNA CAR T cells or a single infusion of $10^{7}$ stably transduced CAR T cells. All anti-CD19 CARs have statistically superior improved median overall survival $(p<0.0001)$ to saline alone, CTX alone, a mesothelin CAR, and the 19-BBz CAR with no interval CTX. There was no significant difference between 19-z, 19-28z, 19-BBz, or 19-28BBz in survival. (B) In this experiment, NSG mice received $10^{6}$ of a primary pediatric high-risk leukemia modified to express the CBG luciferase. Constructs compared include CARs with unmodified sequence cassettes within the CAR or codon optimized versions to reduce internal open reading frames. While the $19-\mathrm{BBz}$ wild type produced the most rapid initial disease response by bioluminescence, the effect was not sustained at the third infusion possibly because too much time had elapsed allowing leukemia to progress. (C) Survival was followed for 126 days, and no statistically significant differences in median overall survival could be appreciated between mRNA constructs $(p=0.22)$. Stably modified CARs were superior in overall survival, with five mice both clearing leukemia and not developing lethal xenogenic GVHD. (D) Day 100 image of mice from the lentiviral CAR groups, demonstrating the jaw/tooth harbor site and the appearance of locoregional relapse.

any lymphodepletion (Fig. 2). In addition, only animals with anti-CD19 RNA CAR T cells showed any benefit, as a combination of antimesothelin RNA CAR T cells and CTX had no effect on disease burden or survival (Fig. 2). This was repeated in separate experiments with two other donors to include cytoxan-only and mock-transduced T-cell controls (Supplementary Fig. S2B and C). A single injection of $10^{7}$ stably transduced LV CAR T cells on day 7 (at 50\% transduction) resulted in rapid and sustained reduction in disease burden, consistent with our historical experience with this model (Milone et al., 2009). A single dose of $60 \mathrm{mg} / \mathrm{kg} \mathrm{IP} \times 1$ of cytoxan was also given to the LV CAR T-cell group on day 27 , in part to investigate if this was able to ameliorate the sometimes lethal xenogeneic GVHD seen at later time points. 
This dose was not sufficient to slow T-cell proliferation at this point, likely because of a significantly higher T-cell burden 20 days after injection (as opposed to 7 days), and no effect on later xenogeneic GVHD was seen. Pseudocolor maps (Fig. 3) reveal that leukemia can be cleared from some sites with subsequent injections but that known xenograft artifact sites such as the tooth root/jaw are resistant to multiple injections and likely serve as the xenograft reservoir for disease progression and relapse once the final injection of RNA CAR T cells has lost receptor expression (Brentjens et al., 2007).

\section{Comparison of CAR costimulatory domains in a multi-infusion model}

We used this model system of repeated infusions of RNA CAR T cells preceded by CTX to evaluate the relative efficacy of first-, second-, and third-generation CAR costimulatory domain structure. Using the $10^{7}-10^{7}-10^{7}(10-10-10)$ repeat infusion dose and schedule, we compared 19-z, 19-28-z, 19$41 \mathrm{BB}-\mathrm{z}, 19-28-41 \mathrm{BB}-\mathrm{z}$ CARs to a meso-41BB-z allogeneic control CAR as well as a saline-only and cytoxan-only control and assessed overall survival in the Nalm-6 CBG model. Although all CD19 CAR constructs were statistically superior to meso-41BB-z and to $19-41 B B-z$ with no CTX $(p<0.001)$, none of the CARs were different from each other in terms of overall survival or median survival (Fig. 4A). We extended this investigation to compare a multi-infusion strategy using either the unmodified sequence of each cassette within the CAR, or codon-optimized RNA of both 1941BB-z and 19-28-41BB-z CARs, comparing them with a single infusion of the same constructs (no codon optimization) expressed stably by LV. For this experiment, we used a CD19+ primary patient sample from a pediatric leukemia that had also been modified with the CBG luciferase (described by Barrett et al., 2011a). Animals were given either a single dose of $10^{7}$ (at 50\% transduction) LV-transduced CAR $+\mathrm{T}$ cells or subjected to the 10-10-10 repeat infusion RNA CAR and CTX schedule. Animals receiving LV-transduced T cells also received a dose of CTX at day 28, in part to investigate if postinfusion lymphodepletion could alleviate the late onset xenogenic GVHD seen in some 1941BB-z-treated animals (>60 days). Consistent with the prior experiment and our previous publications, the most prompt and sustained reduction in bioluminescent disease burden was seen with both lentiviral constructs, followed by the $19-41 \mathrm{BB}-\mathrm{z}$ native sequence RNA CAR T cells (Fig. 4B). Responses to the second T-cell infusion are seen with all RNA CARs, indicating that the lymphodepletion plus T-cell multi-infusion strategy can impact disease burden at multiple time points. Despite initial variation in the impact on disease burden, no single mRNA construct varied significantly in median overall survival, and a single infusion of the stably expressed CARs was superior for survival in both 1928-41BB- $z$ and $19-41 B B-z$ versus the RNA 10-10-10 multiple infusion schedule $\left(10^{7}\right.$ on day $7,10^{7}$ on day 14 , and $10^{7}$ on day 28) ( $p<0.001$; Fig. 4B). As with prior studies, bioluminescent imaging does indicate that subsequent infusions are efficacious against peripheral disease but fare poorly at the jaw/tooth root xenograft harbor site. This serves as an apparently immune privileged reservoir unique to the xenograft that can ultimately lead to progressive total body disease once RNA CAR T-cell infusions are stopped, though we have not observed any CD19-negative variants. This tooth root site also served as the cause of death in two mice from the 19-28-BB-z LV arm (Fig. 4D).

\section{Weighted dose splitting can improve disease control with mRNA CAR T-cell infusions}

In our previous report we achieved significant but temporary disease control with a single high dose $\left(2.5 \times 10^{7}\right)$ of mRNA CAR T cells (Barrett et al., 2011b). We repeated our model of multiple infusions, using $2 \times 10^{7} \mathrm{mRNA}$ CAR T cells on day $7,5 \times 10^{6}$ on day 14 , and $5 \times 10^{6}$ on day $28(20-5-5)$ to see if a high initial dose followed by lower doses could improve on disease control or survival. As there was no superiority for survival demonstrated by the various costimulatory domains, we continued with the $19-41 B B-z$ RNA native sequence construct. While there was no difference in median overall survival (52 vs. 55 days, $p=0.13$ ), we observed complete and sustained disease eradication (out to 130 days) in some animals receiving the 20-5-5 infusion schedule on days 7, 14, and 28 (Fig. 5A). This experiment was repeated four times ( $n=5$ mice per experiment), and multiple infusions produce significant extension in median overall survival compared with a single dose of mRNA CAR T cells $(p=0.01)$. In mice given a total of $3 \times 10^{7} \mathrm{mRNA}$ CAR T cells, a schedule-dependent effect was identified as 20-5-5 was superior to 10-10-10. Furthermore, 20-5-5 was superior to a single large dose of $2.5 \times 10^{7}$.

In addition, mice given a higher initial dose $\left(2 \times 10^{7}\right.$ versus $1 \times 10^{7}$ ) tend to experience more locoregional relapses (similar to the stable expression) rather than systemic relapses as seen with the 10-10-10 schedule (data not shown). A further iteration of this model reduced the infusion schedule to 20 $5-5$ on days 7,14 , and 21 -in essence, a weekly schedule with CTX $24 \mathrm{hr}$ before doses 2 and 3. Two independent experiments (using two different donors) targeting Nalm-6 have resulted in 5/15 mice with complete disease eradication and control for $>120$ days ( 3 mice, donor \#1; 2 mice, donor \#2) and a median overall survival of 80 days versus 55 days for the compressed 20-5-5 schedule (Fig. 5B). The 10 remaining mice succumbed to xenogenic GVHD in ALL remission ( $>60$ days), whereas mice treated with ss1-BBz RNA CAR T cells on this schedule all died of leukemia at $<40$ days. Some mice treated with weekly CTX and irrelevant (mesothelin) RNA CAR T cells survived longer than CTXonly controls (median OS 21 days versus 29 days, $p=0.09$ ). This was seen with donor \#2, and is consistent with a mild alloreactivity of this donor's cells against Nalm-6. Since this effect was not seen with donor \#1, this also suggests that, as intended, the CTX was not itself providing any antileukemic benefit. However, anti-CD19 RNA CAR CTLs demonstrated a significantly more robust effect $(p<0.001)$, including longterm disease control. We repeated these comparisons four times, and the weekly 20-5-5 schedule of infusions results in long-term survival nearly identical to a single infusion of $10^{7}$ stably modified cells (Supplementary Fig. S2).

\section{Discussion}

CAR T-cell therapy is becoming more potent, with better results in clinical trials and better recognition of potential toxicity (Pule et al., 2008; Till et al., 2008; Brentjens et al., 2010, 
A

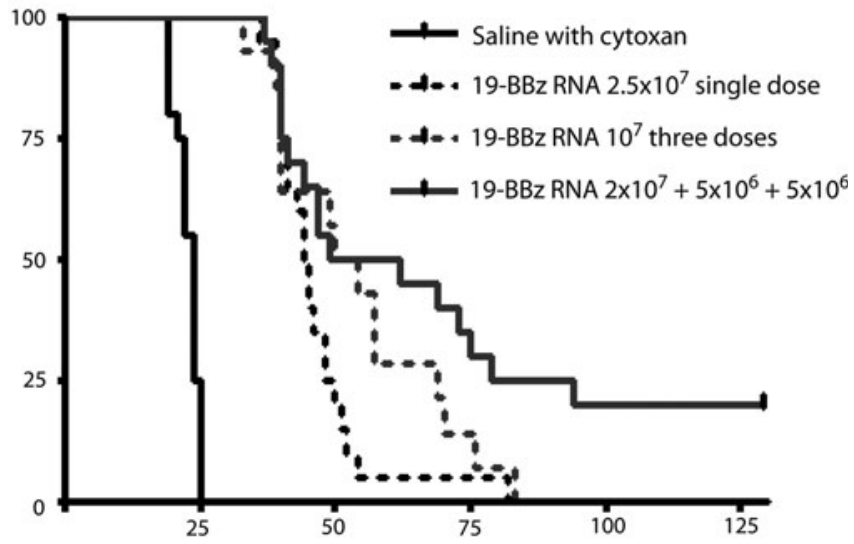

B

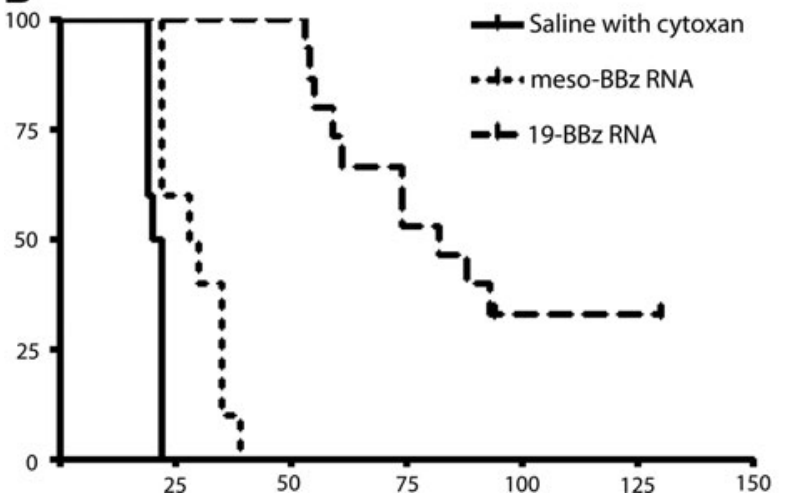

FIG. 5. Dose splitting impacts efficacy of mRNA CAR T cells. (A) This is a composite survival of 4 independent experiments (with 3 different donors, $n=20$ ) using $10^{6}$ Nalm-6 on day 0 and either a single dose $2.5 \times 10^{7}$ on day 7 , or split infusions on days 7,14 , and 28 with interval CTX at $60 \mathrm{mg} /$ $\mathrm{kg} \mathrm{IP} \times 124 \mathrm{hr}$ before CTL doses 2 and 3. Survival is significantly improved by the 20-5-5 schedule despite the total dose of T cells being equivalent ( $p=0.00103)$. The only longterm survivors are observed with this dose schedule. (B) Compressed T-cell infusion schedule further improves efficacy. This composite of 2 experiments with 2 donors demonstrates the significant effect of a compressed infusion schedule of $20-5-5$ on days $7-14-21(n=15, p<0.0001)$. The compressed infusion and CTX schedule did result in some meso-41BBz-treated mice surviving beyond CTX-only controls, perhaps reflecting a slight allogeneic effect of this donor against Nalm-6 (see Supplementary Fig. S2 for further investigation). The difference approached significance for control versus meso-41BBz $(p=0.09)$, while the comparison of control versus anti-CD19-targeted RNA CAR T cells from the same donor was highly significant $(p<0.001)$.

2013; Morgan et al., 2010; Kalos et al., 2011; Porter et al., 2011a; Grupp et al., 2013). By combining a robust T-cell culture system (Levine et al., 1997) that has demonstrated dramatic effects in a clinical trial with the optimized mRNA CAR electroporation protocol and multi-infusion dosing strategy described herein, we have developed a platform that has the potential to increase the therapeutic window with RNA CAR CTLs. The novel finding in this article relates to the unique challenge of delivering systemic CAR therapy for leukemia. While RNA or other temporary modification sys- tems for CARs are widely believed to require repeated infusions to be successful, there has not been a systematic investigation of factors that can make this strategy maximally effective. We demonstrate that, unlike in local delivery models that are not clinically relevant to delivery of antileukemia therapy in patients, a combination of weighted dose splitting and repeat infusions requires interval lymphodepletion to be efficacious. This efficacy is striking, and approaches that of stably modified CAR CTLs in the same models used for their development before clinical success. Given the drug-like kinetics of a temporary CAR, it is perhaps not surprising that a loading dose $\left(2 \times 10^{7}\right)$ followed by lower maintenance doses $\left(5 \times 10^{6}\right)$ at an appropriate half-life interval ( 7 days) results in the best efficacy.

The poor persistence seen in many CAR T-cell trials may be a reflection of the cell manufacturing and subsequent replicative potential of the infused cells. The persistence of the stably transduced CAR T cells in our mouse model may reflect the effectiveness of the infused cells, and cells manufactured in a similar manner but endowed with a temporary CAR via RNA transfection may also be effective in clinical practice. Expression of an anti-CD19 CAR in cytotoxic cells via RNA transfection has now been described by several groups in addition to ours (Rabinovich et al., 2009; Li et al., 2010; Barrett et al., 2011b). Our initial report of the efficacy of anti-CD19 RNA CARs against disseminated leukemia in a preclinical model has now been extended by taking a measured multi-infusion approach. The dose and infusion schedule described here resulted in dramatic responses in both a cell line and a primary patient leukemia sample, advancing the median overall survival in this model closer to that of the stable LV method used in the pilot clinical trials (Porter et al., 2011a,b; Grupp et al., 2013) and providing longterm disease control in some animals.

We chose the timing of the doses (days 7, 14, and 21) based on several iterations of the model that demonstrated that closer spacing (3 days) did not give sufficient time for the current $\mathrm{T}$ cells to finish their effect and that further spacing (14 days) was less effective. In addition, waiting longer than 7 days allowed leukemia in xenograft harbor sites to begin recovery and re-seeding. It is unclear if this jaw/tooth root site is clinically relevant (investigated by Brentjens et al., 2007), and if so RNA CARs may have even more potential clinical efficacy than indicated here. The total of three doses employed in these studies was a practical decision based numbers of available $\mathrm{T}$ cells using our nonclinical-scale manufacturing. It is possible that a larger number of T-cell infusions might have increased the number of animals to achieve long-term disease control. Moving to a weekly schedule increased efficacy the most, including the achievement of long-term durable remissions >100 days since last infusion. The key to success of this approach, however, was the combination of multiple cell infusions with "erasure" of the prior infused cells using lymphodepleting CTX, allowing optimal efficacy of each cell infusion (Cui et al., 2009). Both aspects were required for induction of sustained remissions, and it is important to emphasize that the CTX alone (at the dose employed in our studies) did not provide disease control. Cyclophosphamide may be having other effects other than simple lymphodepletion, including effects on stromal cells or sensitizing effects on residual leukemia. The true mechanism requires further investigation 
beyond the scope of this article, and comparisons to antibody-based depletions (i.e., anti-CD3) and other cytotoxic drugs are warranted. This saturation approach allowed for repeated action of the RNA CAR CTLs over a period consistent with the time needed for disease response in humans seen in the pilot clinical trial. Our data would suggest that the CAR costimulatory domains carry less influence in an RNA model than for an LV model, and that cell dose, interval, and lymphodepleting therapy are the keys to successful RNA CAR efficacy in practical setting of intravenous administration for systemic leukemia. This may reflect the influence of costimulatory domains on long-term persistence of permanently modified CTLs, whereas short-term activation and cytotoxicity are more important with RNA CARs. This would be consistent with our report of cytokine secretion from shortterm cytotoxicity assays assessing each of these costimulatory endodomains, where immediate cytokine release was not significantly different and the primary effect was enhanced long-term proliferation mediated by the 41BB domain (Milone et al., 2009). We were also encouraged by the observations that the third dose again reduced systemic disease, indicating that beneficial effects can be seen even after the leukemia has been exposed to anti-CD19 CARs previously, and we observed no CD19 escape variants in these models.

CARs that incorporate costimulatory domains such as CD28 and $4-1 \mathrm{BB}$ mediate increased cytokine release upon antigen triggering compared with the zeta domain alone, though there is concern that a cytokine release syndrome may be responsible for some adverse events in early CAR trials (Maher et al., 2002; Carpenito et al., 2009; Milone et al., 2009). Temporary CAR expression via RNA, unlike embedded suicide systems, requires no further trigger or systemic treatment to induce $100 \%$ CAR inactivation. While no system could avert some of the immediate toxicity observed in some cases, $\mathrm{T}$ cells can reexpand from a single clone if a suicide system is not $100 \%$ successful. Although still theoretical, the possibility of malignant transformation of a mature $\mathrm{T}$ cell from insertional mutagenesis caused by the retroviral or LV remains a subject of significant regulatory oversight due in no small part to predicted extended lifespan of these cells in children (Nienhuis et al., 2006). As there is no integration into the host cell genome, these regulatory concerns are potentially circumvented by mRNA transfection. In addition, as preclinical mouse models are inadequate for comprehensive safety testing, using RNA CARs in human trials as a first test of a construct or antigen target would allow for an initial safety assessment before administering permanently modified cells. This may be of special importance in solid tumors, when target antigens may be more likely to be present on vital normal tissues. Although the RNA platform would not avoid the immediate-type hypersensitivity or triggered cytokine storms seen in some reported adverse events, it may avoid chronic complications such as the pain seen during infusions of anti-GD2 antibody therapy for neuroblastoma (in the case of anti-GD2 CARs) (Cooper et al., 2004; Yu et al., 2010).

There are several potential limitations of RNA CAR therapy, including the temporary expression, the potential need for greater ex vivo expansion for multiple infusions, and the lack of durable CAR-based antitumor memory cells. The multi-infusion approach, however, brings this platform near the efficacy of stable transfection system in these preclinical models (Carpenito et al., 2009; Milone et al., 2009). RNA CAR
CTLs may be used as a bridge to keep disease burden down while stable CAR CTLs are generated, as well assess safety of that construct in the patient before administration of permanently modified cells. RNA CAR CTLs are self-limited by loss of receptor over time, though this could be augmented by suicide gene systems if necessary (Marktel et al., 2003). As demonstrated in this article, RNA CARs also provide a platform to accelerate the pace of CAR development, providing a flexible and rapid path to the clinic, and thereby enable an efficient iterative approach to optimize CAR design and potency. This is evident within the context of our current study, as seven CAR constructs, four dosing schedules, and addition of interval lymphodepletion were able to be studied in rapid succession without having to generate lentivirus at each stage. RNA CAR CTLs are already being pursued in the clinic, and the multi-infusion and chemotherapy platform described here is informing the design of those trials. In summary, a short-term expression platform that we have developed and refined provides an additional alternative for cell therapy, and we provide some evidence that human RNA CAR CTLs can provide rapid and durable control of CD19+ leukemias. The effectiveness of RNA CAR CTLs regardless of costimulatory domain may point more toward the importance of T-cell manufacture in the success of adoptive immunotherapy, as perhaps the failed persistence was merely a surrogate marker of less effective in vivo $\mathrm{T}$ cells. Although many investigators have hypothesized the need for multiple infusions, the novel aspect of this work lies in highlighting specific requirements for this strategy to have clinical potency in ways not previously studied or suggested such as the weighted dose splitting and timing of lymphodepletion. Defining the T-cell population that may be most effectively modified with CARs, whether temporary or permanent, remains to be determined.

\section{Authorship}

D.M.B. designed the research, performed the experiments, interpreted the data, and wrote the article. X.L. and S.J. performed the research. S.A.G, Y.Z., and C.H.J. designed the research and edited the article.

\section{Acknowledgments}

This work was supported in part by research grants R01CA120409 and P01CA066726 (C.H.J. and Y.Z.), 2-K12CA-076931-11 (D.M.B.), and R01CA102646 and Pennsylvania Department of Health (S.A.G.), as well as by Weinberg, Cookies for Kids, and W.W. Smith Foundations (S.A.G.).

\section{Author Disclosure Statement}

Y.Z. and C.H.J. have patent applications and intellectual property in some of the technology described in this article. The other authors have no conflict of interest.

\section{References}

Barrett, D.M., Seif, A.E., et al. (2011a). Noninvasive bioluminescent imaging of primary patient acute lymphoblastic leukemia: a strategy for preclinical modeling. Blood 118, e112-e117.

Barrett, D.M., Zhao, Y., et al. (2011b). Treatment of advanced leukemia in mice with mRNA engineered T cells. Hum. Gene Ther. 22, 1575-1586. 
Bleyer, W.A. (1985). Cancer chemotherapy in infants and children. Pediatr. Clin. North Am. 32, 557-574.

Bracci, L., Moschella, F., et al. (2007). Cyclophosphamide enhances the antitumor efficacy of adoptively transferred immune cells through the induction of cytokine expression, B-cell and T-cell homeostatic proliferation, and specific tumor infiltration. Clin. Cancer Res. 13(2 Pt 1), 644-653.

Brentjens, R.J., Santos, E., et al. (2007). Genetically targeted T cells eradicate systemic acute lymphoblastic leukemia xenografts. Clin. Cancer Res. 13(18 Pt 1), 5426-5435.

Brentjens, R., Yeh, R., et al. (2010). Treatment of chronic lymphocytic leukemia with genetically targeted autologous $\mathrm{T}$ cells: case report of an unforeseen adverse event in a phase I clinical trial. Mol. Ther. 18, 666-668.

Brentjens, R.J., Riviere, I., et al. (2011). Safety and persistence of adoptively transferred autologous CD19-targeted T cells in patients with relapsed or chemotherapy refractory B-cell leukemias. Blood 118, 4817-4828.

Brentjens, R.J., Davila, M.L., et al. (2013). CD19-targeted $\mathrm{T}$ cells rapidly induce molecular remissions in adults with chemotherapy-refractory acute lymphoblastic leukemia. Sci. Transl. Med. 5, 177ra38.

Brocker, T., and Karjalainen, K. (1998). Adoptive tumor immunity mediated by lymphocytes bearing modified antigenspecific receptors. Adv. Immunol. 68, 257-269.

Carpenito, C., Milone, M.C., et al. (2009). Control of large, established tumor xenografts with genetically retargeted human $\mathrm{T}$ cells containing CD28 and CD137 domains. Proc. Natl. Acad. Sci. USA 106, 3360-3365.

Cooper, L.J., Al-Kadhimi, Z., et al. (2004). Development and application of CD19-specific $\mathrm{T}$ cells for adoptive immunotherapy of B cell malignancies. Blood Cells Mol. Dis. 33, 83-89.

Cui, Y., Zhang, H., et al. (2009). Harnessing the physiology of lymphopenia to support adoptive immunotherapy in lymphoreplete hosts. Blood 114, 3831-3840.

Elkin, G., Prigozhina, T.B., et al. (2004). Prevention of diabetes in nonobese diabetic mice by nonmyeloablative allogeneic bone marrow transplantation. Exp. Hematol. 32, 579-584.

Eshhar, Z., Waks, T., et al. (1993). Specific activation and targeting of cytotoxic lymphocytes through chimeric single chains consisting of antibody-binding domains and the gamma or zeta subunits of the immunoglobulin and T-cell receptors. Proc. Natl. Acad. Sci. USA 90, 720-724.

Gammon, S.T., Leevy, W.M., et al. (2006). Spectral unmixing of multicolored bioluminescence emitted from heterogeneous biological sources. Anal. Chem. 78, 1520-1527.

Grupp, S.A., Kalos, M., et al. (2013). Chimeric antigen receptormodified T cells for acute lymphoid leukemia. N. Engl. J. Med. 368, 1509-1518.

Horowitz, M.M., Gale, R.P., et al. (1990). Graft-versus-leukemia reactions after bone marrow transplantation. Blood 75, 555562.

Imai, C., Mihara, K., et al. (2004). Chimeric receptors with 4-1BB signaling capacity provoke potent cytotoxicity against acute lymphoblastic leukemia. Leukemia 18, 676-684.

Kalos, M., Levine, B., et al. (2011). T cells with chimeric antigen receptors have potent antitumor effects and can establish memory in patients with advanced leukemia. Sci. Transl. Med. 3, 95ra73.

Kershaw, M.H., Westwood, J.A., et al. (2006). A phase I study on adoptive immunotherapy using gene-modified $\mathrm{T}$ cells for ovarian cancer. Clin. Cancer Res. 12(20 Pt 1), 6106-6115.

Kochenderfer, J.N., Yu, Z., et al. (2010a). Adoptive transfer of syngeneic $T$ cells transduced with a chimeric antigen receptor that recognizes murine CD19 can eradicate lymphoma and normal B cells. Blood 116, 3875-3886.

Kochenderfer, J.N., Wilson, W.H., et al. (2010b). Eradication of Blineage cells and regression of lymphoma in a patient treated with autologous $\mathrm{T}$ cells genetically engineered to recognize CD19. Blood 116, 4099-4102.

Lee, J.C., Hayman, E., et al. (2011). In vivo inhibition of human CD19-targeted effector $\mathrm{T}$ cells by natural $\mathrm{T}$ regulatory cells in a xenotransplant murine model of B cell malignancy. Cancer Res. 71, 2871-2881.

Levine, B., Bernstein, W., et al. (1997). Effects of CD28 costimulation on long-term proliferation of CD4 + T cells in the absence of exogenous feeder cells. J. Immunol. 159, 5921-5930.

Li, L., Liu, L.N., et al. (2010). Expression of chimeric antigen receptors in natural killer cells with a regulatory-compliant non-viral method. Cancer Gene Ther. 17, 147-154.

Lu, Y., Douraghy, A., et al. (2009). Spectrally resolved bioluminescence tomography with the third-order simplified spherical harmonics approximation. Phys. Med. Biol. 54, 6477-6493.

Maher, J., Brentjens, R.J., et al. (2002). Human T-lymphocyte cytotoxicity and proliferation directed by a single chimeric TCR zeta/CD28 receptor. Nat. Biotechnol. 20, 70-75.

Malone, R.W., Felgner, P.L., et al. (1989). Cationic liposomemediated RNA transfection. Proc. Natl. Acad. Sci. USA 86, 6077-6081.

Mao, Y. (2011). Dynamics studies of luciferase using elastic network model: how the sequence distribution of luciferase determines its color. Protein Eng. Des. Sel. 24, 341-349.

Marktel, S., Magnani, Z., et al. (2003). Immunologic potential of donor lymphocytes expressing a suicide gene for early immune reconstitution after hematopoietic T-cell-depleted stem cell transplantation. Blood 101, 1290-1298.

Masters, J.R., Thomson, J.A., et al. (2001). Short tandem repeat profiling provides an international reference standard for human cell lines. Proc Natl Acad Sci USA 98, 8012-8017.

Mezzanotte, L., Que, I., et al. (2011). Sensitive dual color in vivo bioluminescence imaging using a new red codon optimized firefly luciferase and a green click beetle luciferase. PLoS One 6, e19277.

Milone, M.C., Fish, J.D., et al. (2009). Chimeric receptors containing CD137 signal transduction domains mediate enhanced survival of $\mathrm{T}$ cells and increased antileukemic efficacy in vivo. Mol. Ther. 17, 1453-1464.

Morgan, R.A., Yang, J.C., et al. (2010). Case report of a serious adverse event following the administration of T cells transduced with a chimeric antigen receptor recognizing ERBB2. Mol. Ther. 18, 843-851.

Nadler, L.M., Anderson, K.C., et al. (1983). B4, a human B lymphocyte-associated antigen expressed on normal, mitogenactivated, and malignant B lymphocytes. J. Immunol. 131, 244-250.

Nakajima, Y., Kimura, T., et al. (2005). Multicolor luciferase assay system: one-step monitoring of multiple gene expressions with a single substrate. Biotechniques 38, 891-894.

Nienhuis, A.W., Dunbar, C.E., et al. (2006). Genotoxicity of retroviral integration in hematopoietic cells. Mol. Ther. 13, 10311049.

Pinthus, J.H., Waks, T., et al. (2004). Adoptive immunotherapy of prostate cancer bone lesions using redirected effector lymphocytes. J. Clin. Invest. 114, 1774-1781.

Porter, D.L., Levine, B.L., et al. (2011a). Chimeric antigen receptor-modified $\mathrm{T}$ cells in chronic lymphoid leukemia. N. Engl. J. Med. 365, 725-733. 
Pule, M.A., Savoldo, B., et al. (2008). Virus-specific T cells engineered to coexpress tumor-specific receptors: persistence and antitumor activity in individuals with neuroblastoma. Nat. Med. 14, 1264-1270.

Rabinovich, P.M., Komarovskaya, M.E., et al. (2009). Chimeric receptor mRNA transfection as a tool to generate antineoplastic lymphocytes. Hum. Gene Ther. 20, 51-61.

Savoldo, B., Ramos, C.A., et al. (2011). CD28 costimulation improves expansion and persistence of chimeric antigen receptor-modified $\mathrm{T}$ cells in lymphoma patients. J. Clin. Invest. $121,1822-1826$.

Shimasaki, N., Fujisaki, H., et al. (2012). A clinically adaptable method to enhance the cytotoxicity of natural killer cells against B-cell malignancies. Cytotherapy 14, 830-840.

Tafreshi, N., Sadeghizadeh, M., et al. (2008). Site-directed mutagenesis of firefly luciferase: implication of conserved residue(s) in bioluminescence emission spectra among firefly luciferases. Biochem. J. 412, 27-33.

Teachey, D.T., Obzut, D.A., et al. (2006). The mTOR inhibitor CCI-779 induces apoptosis and inhibits growth in preclinical models of primary adult human ALL. Blood 107, 1149-1155.

Till, B.G., Jensen, M.C., et al. (2008). Adoptive immunotherapy for indolent non-Hodgkin lymphoma and mantle cell lymphoma using genetically modified autologous CD20-specific $\mathrm{T}$ cells. Blood 112, 2261-2271.

Till, B.G., Jensen, M.C., et al. (2012). CD20-specific adoptive immunotherapy for lymphoma using a chimeric antigen receptor with both CD28 and 4-1BB domains: pilot clinical trial results. Blood 119, 3940-3950.
Yu, A.L., Gilman, A.L., et al. (2010). Anti-GD2 antibody with GM-CSF, interleukin-2, and isotretinoin for neuroblastoma. N. Engl. J. Med. 363, 1324-1334.

Zhao, Y., Zheng, Z., et al. (2005). Primary human lymphocytes transduced with NY-ESO-1 antigen-specific TCR genes recognize and kill diverse human tumor cell lines. J. Immunol. $174,4415-4423$.

Zhao, Y., Zheng, Z., et al. (2006). High-efficiency transfection of primary human and mouse T lymphocytes using RNA electroporation. Mol. Ther. 13, 151-159.

Zhao, Y., Moon, E., et al. (2010). Multiple injections of electroporated autologous $\mathrm{T}$ cells expressing a chimeric antigen receptor mediate regression of human disseminated tumor. Cancer Res. 70, 9053-9061.

Address correspondence to: Dr. Stephan A. Grupp Division of Oncology and Department of Pathology Children's Hospital of Philadelphia Colket Translational Research Building Room 3006

3501 Civic Center Boulevard Philadelphia, PA 19104

E-mail: grupp@email.chop.edu

Received for publication April 12, 2013;

accepted after revision July 17, 2013.

Published online: July 24, 2013. 